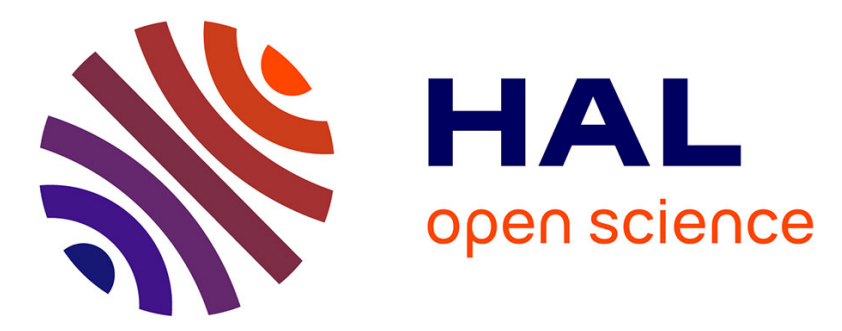

\title{
Epitaxial ZnO thin films grown by pulsed electron beam deposition
}

\author{
Sylvain Tricot, Magdalena Nistor, Éric Millon, Chantal Boulmer-Leborgne, \\ Nicolae Bogdan Mandache, Jacques Perriére, Wilfried Seiler
}

\section{- To cite this version:}

Sylvain Tricot, Magdalena Nistor, Éric Millon, Chantal Boulmer-Leborgne, Nicolae Bogdan Mandache, et al.. Epitaxial $\mathrm{ZnO}$ thin films grown by pulsed electron beam deposition. Surface Science: A Journal Devoted to the Physics and Chemistry of Interfaces, 2010, 604 (21-22), pp.2024-2030. 10.1016/j.susc.2010.08.016 . hal-02269228

\section{HAL Id: hal-02269228 https://hal.science/hal-02269228}

Submitted on 22 Aug 2019

HAL is a multi-disciplinary open access archive for the deposit and dissemination of scientific research documents, whether they are published or not. The documents may come from teaching and research institutions in France or abroad, or from public or private research centers.
L'archive ouverte pluridisciplinaire HAL, est destinée au dépôt et à la diffusion de documents scientifiques de niveau recherche, publiés ou non, émanant des établissements d'enseignement et de recherche français ou étrangers, des laboratoires publics ou privés. 


\title{
Epitaxial ZnO thin films grown by pulsed electron beam deposition
}

\author{
S. Tricot ${ }^{\text {a,* }}$, M. Nistor ${ }^{\text {b }}$, E. Millon ${ }^{\text {a }}$, C. Boulmer-Leborgne ${ }^{\text {a }}$, N.B. Mandache ${ }^{\text {b }}$, J. Perrière ${ }^{c}$, W. Seiler ${ }^{d}$ \\ a GREMI, UMR 6606 CNRS/Université d'Orléans, 14 rue d'Issoudun, B.P. 6744, 45067 Orléans Cedex 2, France \\ b National Institute for Lasers, Plasma and Radiation Physics, PO Box MG-36, Bucharest-Magurele, Romania \\ c INSP, UMR 7588 CNRS/Université Pierre et Marie Curie - Paris 6, 140 rue de Lourmel, 75015 Paris, France \\ ' PIMM, UMR 8006 CNRS/ENSAM, 151 Boulevard de l'Hôpital, 75013 Paris, France
}

Keywords:

Pulsed electron beam deposition (PED)

Zinc oxide

Pole figure

Resistivity

Tauc plot

\begin{abstract}
A B S T R A C T
In this work, the pulsed electron beam deposition method (PED) is evaluated by studying the properties of $\mathrm{ZnO}$ thin films grown on c-cut sapphire substrates. The film composition, structure and surface morphology were investigated by means of Rutherford backscattering spectrometry, X-ray diffraction and atomic force microscopy. Optical absorption, resistivity and Hall effect measurements were performed in order to obtain the optical and electronic properties of the $\mathrm{ZnO}$ films. By a fine tuning of the deposition conditions, smooth, dense, stoichiometric and textured hexagonal $\mathrm{ZnO}$ films were epitaxially grown on (0001) sapphire at $700{ }^{\circ} \mathrm{C}$ with a $30^{\circ}$ rotation of the $\mathrm{ZnO}$ basal plane with respect to the sapphire substrate. The average transmittance of the films reaches $90 \%$ in the visible range with an optical band gap of $3.28 \mathrm{eV}$. Electrical characterization reveals a high density of charge carrier of $3.4 \times 10^{19} \mathrm{~cm}^{-3}$ along with a mobility of $11.53 \mathrm{~cm}^{2} / \mathrm{Vs}$. The electrical and optical properties are discussed and compared to $\mathrm{ZnO}$ thin films prepared by the similar and most well-known pulsed laser deposition method.
\end{abstract}

\section{Introduction}

Pulsed electron beam deposition (PED) is a recent and simple method for deposition of thin films. In PED, a pulsed electron beam is used to ablate the surface of a target material and the emitted species are collected onto a substrate in front of the target [1,2]. The PED setup is thus very similar to the pulsed laser deposition technique (PLD).

In a previous paper we showed that whatever the nature, composition and properties of the materials grown by PED, particulates of the order of tens-hundred of $\mathrm{nm}$ in diameter are present at the surface of the thin films, but by a careful optimization of the electron beam parameters it is possible to drastically reduce the density and size of particulates at the surface of the films [2]. This could be explained by a unique feature of the pulsed-beam which delivers electrons with a large energy distribution, with a dominant highenergy electron component at earlier times and a contribution of lower energy electrons increasing towards later times [3-5].

The investigation of the plasma plume produced by the pulsed electron beam with the target by fast imaging and optical emission spectroscopy $[3,5]$ showed that the kinetic energies of the species emitted by the target is about $10-50 \mathrm{eV}$, thus enhancing the adatom mobility when arriving on the substrate surface and providing enough energy to enhance the film growth while avoiding surface droplets.

\footnotetext{
* Corresponding author.

E-mail address: sylvain.tricot@univ-rennes1.fr (S. Tricot).
}

One interesting aspect of the PED method is its ability to ablate a wide range of target materials and especially wide band gap semiconductors that are more difficult to process by PLD since they are poorly absorbent to the laser light. Zinc oxide $(\mathrm{ZnO})$ is an example of such materials and it has already been shown that ZnO thin films grown by PLD are of better quality when prepared using a laser wavelength shorter than the band gap of $\mathrm{ZnO}$ [6]. Thus, PED could be an alternative to PLD for large band gap materials.

Moreover, $\mathrm{ZnO}$ has very attractive properties. It is a natural n-type semiconductor with resistivity values ranging from 10 to $10^{-2} \Omega / \mathrm{cm}$ [7] for undoped $\mathrm{ZnO}$. Optical properties of $\mathrm{ZnO}$ also focus intense research due to its wide direct band gap of $3.3 \mathrm{eV}$ which makes $\mathrm{ZnO}$ transparent to the visible light. Its properties makes therefore $\mathrm{ZnO}$ promising for applications in optoelectronics, piezoelectric devices, SAW filters, gas sensors, solar cell transparent electrodes, transparent electronics and spintronics [7-9]. Some of these applications require very well-crystallized $\mathrm{ZnO}$ thin films or even $\mathrm{ZnO}$ epitaxially grown on a substrate. However, the growth of $\mathrm{ZnO}$ films by PLD is now well optimized [10] but despite the large number of data already reported in the literature on the structural and physical properties of ZnO films grown under various experimental conditions, very few studies give an accurate description of ZnO films grown by PED [11].

In this work, we investigate the morphological, structural, optical and electrical properties of $\mathrm{ZnO}$ thin films prepared by PED. The aim is to compare these films with known results from the literature of PLD prepared $\mathrm{ZnO}$ films to know if PED could be a reliable alternative to PLD for wide band gap materials. To hold a comparison of both techniques and to be only sensitive to the deposition process, growths 
were performed on sapphire $(000 l)$ substrate held at $700{ }^{\circ} \mathrm{C}$ without any post-treatment. These conditions - classically reported in PLD are known to give well crystallized and epitaxied films.

\section{Experimental}

ZnO thin films were grown by the PED method on c-cut sapphire (0001) single crystal substrates. The experimental set-up of PED, described in detail in previous papers [2,5] is sketched in Fig. 1. Basically, a pulsed-electron beam interacts with a solid ZnO target and induces a plasma. The ablated species are collected onto the substrate facing the target and a thin film is formed pulses after pulses much like the pulsed laser deposition technique. The produced electron beam pulse width is $100 \mathrm{~ns}$ (FWHM), the total current reaches $800 \mathrm{~A}$ and it is composed of electrons with energies ranging from a few $\mathrm{eV}$ to the energy corresponding to the applied high voltage (15 keV for an applied voltage of $-15 \mathrm{kV}$ for example). The beam diameter is in the $2-4 \mathrm{~mm}$ range leading to a fluence of about $2.5 \mathrm{~J} / \mathrm{cm}^{2}$. The electron beam source consists of a hollow cathode, a dielectric capillary tube (6 $\mathrm{mm}$ in diameter and $110 \mathrm{~mm}$ in length) and the vacuum chamber as grounded anode. At each pulse, a $16 \mathrm{nF}$ external capacitor is discharged between the cathode and the anode. The discharge voltage was varied in the $14-16 \mathrm{kV}$ range. The maximum pulse repetition rate of the device is $5 \mathrm{~Hz}$ and was kept at $1 \mathrm{~Hz}$ for these experiments. The target-substrate distance is set to $40 \mathrm{~mm}$ and the substrate was heated at $700{ }^{\circ} \mathrm{C}$. Indeed growth temperatures higher than $300{ }^{\circ} \mathrm{C}$ is known to favor a better crystallinity of films [7]. During the film growth the argon pressure was kept at $1.4 \times 10^{-2}$ mbar inside the chamber. This argon pressure is needed for the electron beam operation. After the growth, films are cooled down in the ablation chamber under the same argon pressure.

Rutherford backscattering spectrometry, using $2 \mathrm{MeV}$ energy ${ }^{4} \mathrm{He}^{+}$ ion beams from the Van de Graaff accelerator of the Institut des NanoSciences de Paris (INSP-Université Paris 6) was used to measure the thickness and composition of the zinc oxide films. The accurate determination of the in-depth distribution of the elements in films was obtained by the RUMP simulation program [12]. Due to the low RBS yield on oxygen, the relative accuracy of this element in films was therefore close to $4 \%$. The surface morphology of films was investigated by atomic force microscopy. The structural characterizations of the zinc oxide films were carried out by XRD analyses using a

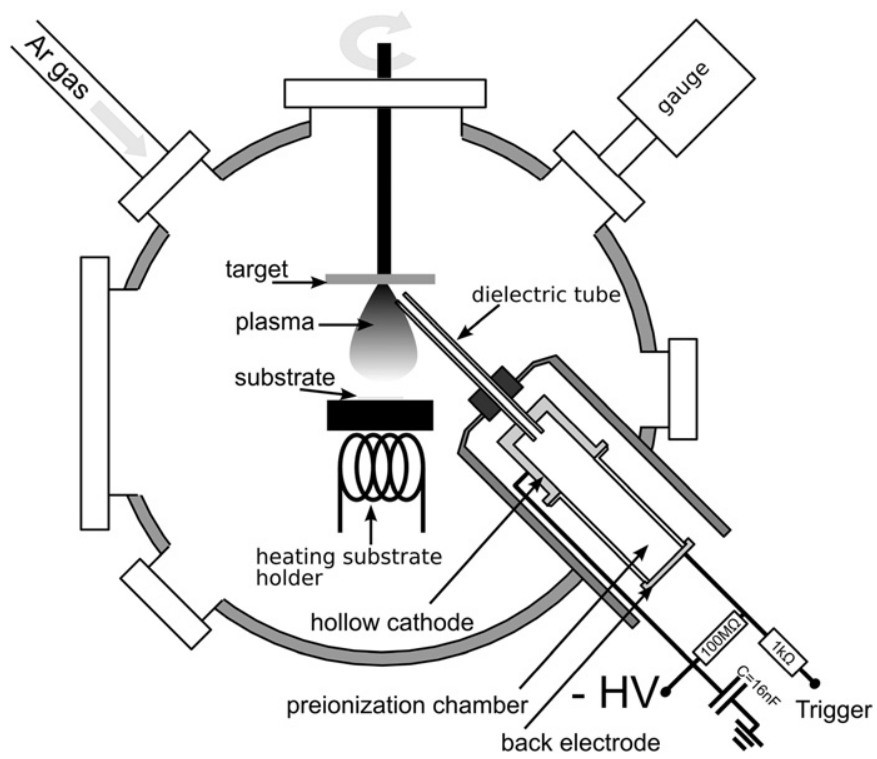

Fig. 1. Top view of the PED experimental setup. The dielectric tube defines an angle of $45^{\circ}$ with the target. four circle diffractometer (Philips Xpert MRD of LIM laboratoryENSAM-Paris) with the $\mathrm{Cu} K \alpha$ radiation $(\lambda=0.154 \mathrm{~nm})$. The nature of the crystalline phases, their axis parameters and their preferred orientations with respect to the substrates were investigated by XRD experiments in the Bragg-Brentano geometry. The in-plane epitaxial relationships between films and single crystal substrates were studied by asymmetric diffraction with the measurements of pole figures of selected reflection peaks of the film and substrates. The optical absorption of films was measured in the $200-2500 \mathrm{~nm}$ range with a Cary 5000 spectrophotometer. To obtain a better understanding of the properties of excitons in $\mathrm{ZnO}$ thin films, photoluminescence measurements were carried out at room temperature using a xenon lamp as excitation source at $\lambda=350 \mathrm{~nm}$. The transport properties (resistivity as a function of temperature from room temperature down to liquid He temperature) of the zinc oxide films were obtained by the classical four probe method. The carrier type and concentration as well as their mobility were determined with a MMR Hall measurement system in the Van der Pauw geometry at a magnetic field of $0.3 \mathrm{~T}$.

\section{Results and discussion}

\subsection{Film composition and morphology}

Parameters of the pulsed-electron beam for the growth of zinc oxide films were optimized in order to obtain the formation of dense and smooth zinc oxide films free of particulates. The RBS spectra recorded on such zinc oxide films grown on $c$-cut sapphire substrate at $700{ }^{\circ} \mathrm{C}$ evidenced that no interdiffusion occurs between the film and the substrate and that the surface roughness is smooth. A uniform indepth distribution of $\mathrm{Zn}$ and $\mathrm{O}$ species was also observed in the films, leading to the conclusion that nearly stoichiometric $\mathrm{ZnO}$ films are formed in these conditions. Moreover, argon incorporation in the zinc oxide films was not evidenced despite the fact these films were grown under a relatively high argon partial pressure $\left(1.4 \times 10^{-2} \mathrm{mbar}\right)$. This point is one of the main specificity of the PED growth method with respect to other growth methods like conventional sputtering which lead to argon incorporation in the films during growth under similar argon pressure [13]. In PED, growth occurs in presence of an argon plasma whose species (Ar ions or atoms) have a low kinetic energy (maximum a few eV). Such species can be physically adsorbed at the surface or in the near surface region of the growing film. Meanwhile, species directly emitted by the target during and after the electron bombardment fly to the substrate with large kinetic energies (in the tens of $\mathrm{eV}$ range) [2,5]. In these conditions, the bombardment of the film surface with the energetic target species induces the desorption of any weakly adsorbed species like Ar which could be incorporated. Thus, the ZnO films grown by PED are free from argon. It is worth noticing that $\mathrm{ZnO}$ films prepared by PLD are usually grown under an oxygen partial pressure that can be varied in a wide range from $10^{-5}$ to $10^{-1}$ mbar. Without addition of oxygen during the growth, the resulting films are zinc enriched. Thanks to the optimization of the discharge parameters, we showed here that it is possible to obtain nearly stoichiometric $\mathrm{ZnO}$ thin film by PED without adding oxygen in the chamber.

It is also important to note that the growth rate of the PED technique is in the $0.05-0.11 \mathrm{~nm} /$ pulse range which is more than 10 times greater than those classically obtained by PLD. This feature is an interesting characteristic of the PED process as the growth rate is known to be an experimental parameter limiting a broader use of the PLD technique for industrial trends.

According to atomic force microscopy experiments, the surface of ZnO films grown by PED is smooth with a mean roughness of $4.9 \mathrm{~nm}$ (RMS roughness of $7.9 \mathrm{~nm}$ ) for a total thickness of $200 \mathrm{~nm}$. The boundaries of the crystallites are also visible and their diameter can be estimated to $40 \mathrm{~nm}$. 


\subsection{Film structure}

The crystallization of the $\mathrm{ZnO}$ thin films on c-cut sapphire substrate was studied for the fixed experimental growth conditions leading to the growth of dense, smooth and stoichiometric films. The XRD diagram in the Bragg-Brentano geometry of $\mathrm{ZnO}$ thin films grown by PED on a $c$-cut sapphire substrate at $700{ }^{\circ} \mathrm{C}$ is shown in Fig. 2. A strong well-defined peak at $2 \theta=34.375^{\circ}$ as well as a weak peak around $72^{\circ}$ are observed on this diagram. It corresponds respectively to the (0002) and the (0004) diffraction peaks of the $\mathrm{ZnO}$ hexagonal wurtzite structure, indicating that the $\mathrm{ZnO}$ thin films are grown with the $c$ axis normal to the sapphire substrate plane. This c-axis orientation of crystallites is classically observed for $\mathrm{ZnO}$ films whatever the growth method. Indeed, textured films are generally obtained when the plane parallel to the substrate has the lowest surface energy density [14]. In the case of $\mathrm{ZnO}$ which is a polar crystal (alternative stacking of $\mathrm{Zn}$ planes and $\mathrm{O}$ planes), the surface free energy of the (000l) plane has not a finite value. To limit the surface of such plane, the growth rate along the $c$-axis is increased and thus a columnar growth of $\mathrm{ZnO}$ crystallites is evidenced. The value of the $c$ axis lattice parameter deduced from the diffraction pattern is $c=0.52125 \mathrm{~nm}$. This value nearly reaches the bulk $\mathrm{ZnO}$ value $c_{\text {bulk }}=0.52066 \mathrm{~nm}$. The estimated size of the $\mathrm{ZnO}$ crystallites through the Debye-Scherrer formula is $44 \mathrm{~nm}$. The crystallite size in $\mathrm{ZnO}$ films grown by PLD is typically about $50 \mathrm{~nm}$ with the same columnar growth on sapphire [15].

The mosaic spread of this (0002) texture was studied by rocking curve measurements. The inset in Fig. 2 represents the (0002) rocking curve of the $\mathrm{ZnO}$ films on c-cut sapphire substrate. The FWHM of the rocking curve is only of $0.65^{\circ}$ evidencing therefore a low mosaic spread of the crystallites according to the main axis growth and a high crystalline quality of the film similar to that of obtained in PLD films.

The (0002) texture observed in $\mathrm{ZnO}$ films suggests a possible epitaxial growth of the films. Pole figure measurements were performed to investigate the epitaxial relationships between the $\mathrm{ZnO}$ film and the sapphire substrate. Fig. 3 reports pole figures recorded for the $\{10 \overline{1} 3\} \mathrm{ZnO}$ family planes $\left(2 \theta=62.86^{\circ}\right)$. Six well defined poles at a declination angle $\Psi=32.5^{\circ}$ are visible, indicating the existence of an epitaxial relationship between the film and the substrate. Moreover, three poles at a declination angle $\Psi=22.5^{\circ}$ are also observed, corresponding to the poles of the $\{01 \overline{1} 8\}$ planes of the c-cut sapphire substrate $\left(2 \theta=61.3^{\circ}\right)$.
Considering the sapphire substrate symmetry, only three poles should be observed for the $\{10 \overline{1} 3\}$ family planes of $\mathrm{ZnO}$. This has already been observed and explained by the fact that the sapphire surface exhibit steps which induce an unconventional number of poles ( 6 poles $60^{\circ}$ apart) and a symmetry of order 6 instead of 3 [16].

The comparison of the position of the six poles of the $\{10 \overline{1} 3\} \mathrm{ZnO}$ planes, with the poles of the $\{01 \overline{1} 8\}$ family planes of the sapphire substrate, evidences a rotation of $30^{\circ}$ of the $\mathrm{ZnO}$ axis with respect to those of the c-cut sapphire substrate. The following orientation relationship can therefore be inferred:

$$
\mathrm{ZnO}(0001)[2 \overline{1} \overline{1} 0] / / \mathrm{Al}_{2} \mathrm{O}_{3}(0001)[1 \overline{1} 00]
$$

Such an epitaxial growth is classically observed for the growth of $\mathrm{ZnO}$ thin films on $c$-cut sapphire substrates [17]. The $30^{\circ}$ rotation between the 2 cells ensures the continuity of the hexagonal oxygen sublattice in the sapphire substrate and in the $\mathrm{ZnO}$ film. These orientations can be described in terms of "domain matching epitaxy" [18], which has been already used to describe the same epitaxial relationships evidenced in PLD ZnO films grown on sapphire at $700{ }^{\circ} \mathrm{C}$ [15].

In summary, regarding the main axis growth of the $\mathrm{ZnO}$ crystallites, their in-plane orientations with the sapphire substrate, and their estimated size in the several tens of nm range, the PED technique leads to ZnO films having a crystalline quality very similar to those obtained by PLD at the same substrate temperature.

\subsection{Electrical measurements}

The transport properties of the $\mathrm{ZnO}$ thin films grown by PED on $c$ cut sapphire substrates were investigated by resistivity and Hall effect measurements. The normalized resistivity as a function of the temperature is reported in Fig. 4. The resistivity decreases while the temperature increases revealing the semiconducting behavior of the thin film in the whole temperature range as it is classically observed with PLD ZnO films.

Hall effect measurements were performed at room temperature. It is found that the $\mathrm{ZnO}$ film is $n$-type and has a high amount of charge carriers $\left(n_{\mathrm{e}}=3.4 \times 10^{19} \mathrm{~cm}^{-3}\right)$. This is a very high value compared to undoped $\mathrm{ZnO}$ thin films prepared by PLD which are usually in the $10^{16} \mathrm{~cm}^{-3}$ range [7]. However, the electron mobility is only of $11.53 \mathrm{~cm}^{2} / \mathrm{Vs}$ at room temperature. This value of $\mu_{\mathrm{e}}$ is far from the

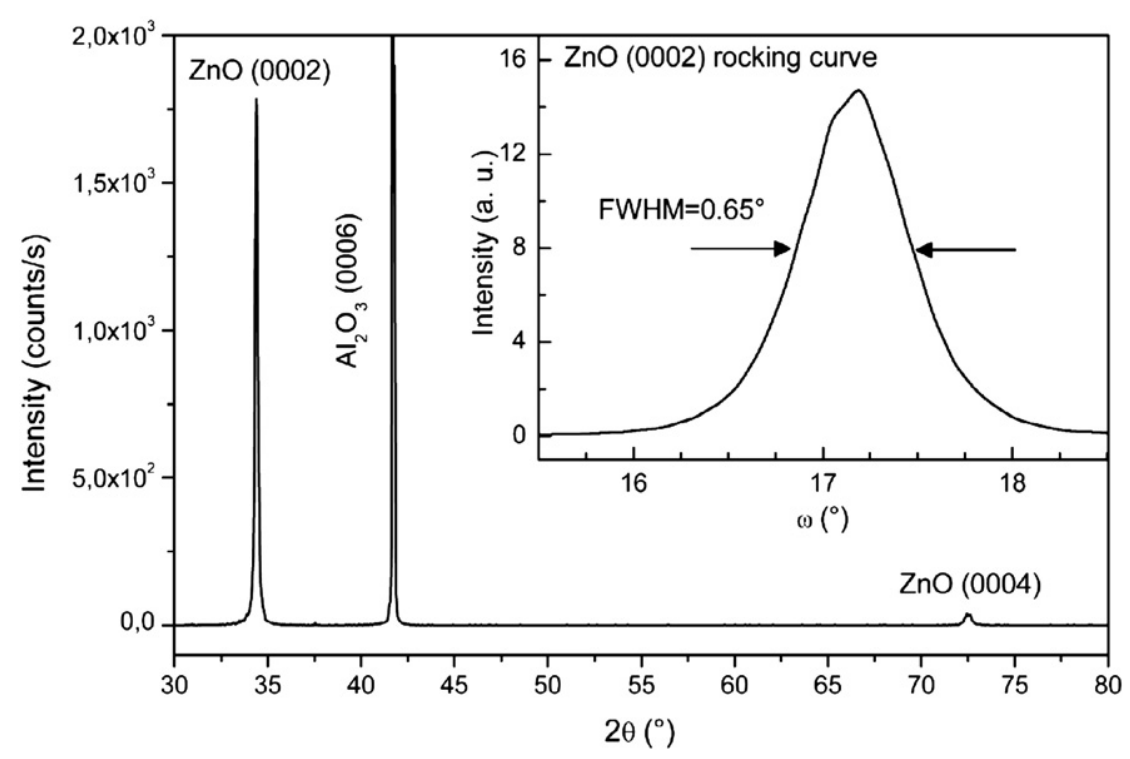

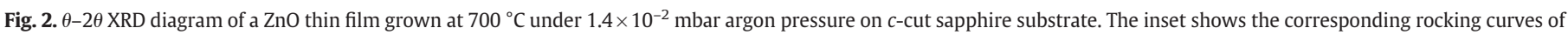
the (0002) reflection. 


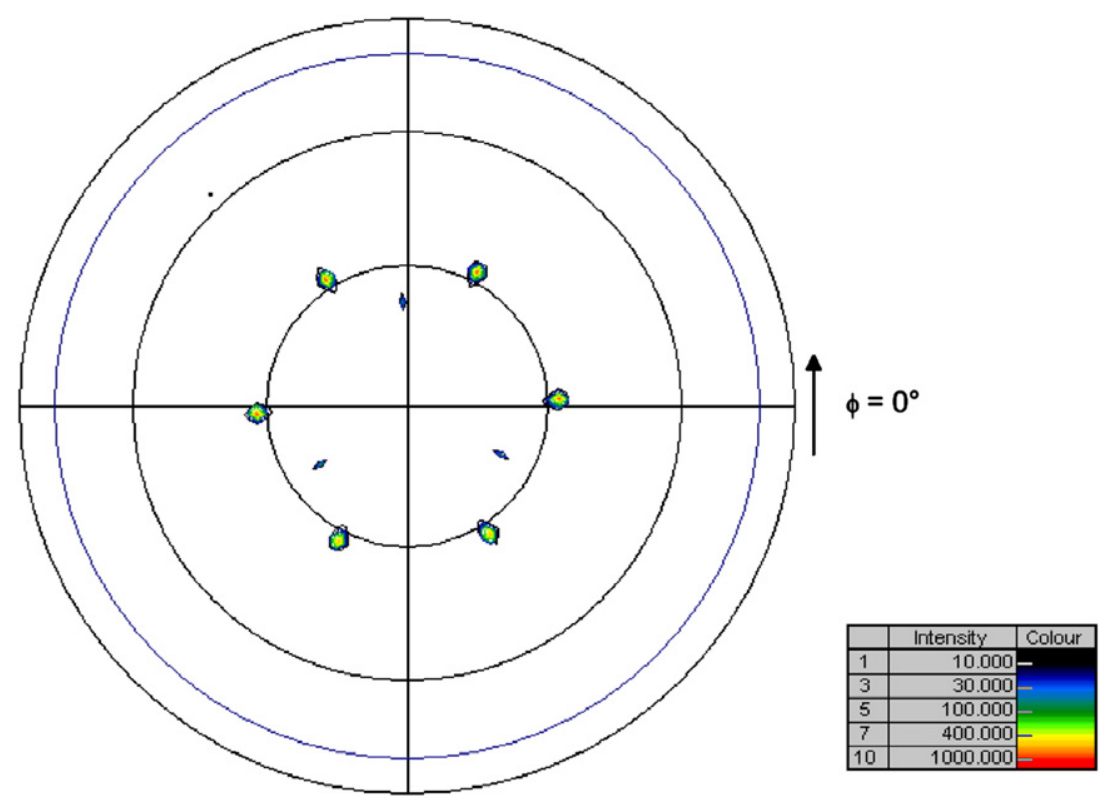

Fig. 3. $\mathrm{Al}_{2} \mathrm{O}_{3}(01 \overline{1} 8)$ and $\mathrm{ZnO}(10 \overline{1} 3)$ pole figure of a zinc oxide film grown by PED at $700{ }^{\circ} \mathrm{C}$ on a $c$-cut sapphire substrate.

expected theoretical value of $300 \mathrm{~cm}^{2} /$ vs for the bulk single crystal [19]. Thanks to the high density of charge carriers, the resistivity at room temperature is as low as $1.6 \times 10^{-2} \Omega \mathrm{cm}$. The good crystalline quality of the PED films and their stoichiometry (low concentration of oxygen vacancies) are in good agreement with a resistivity around $10^{-2} \Omega \mathrm{cm}$. This value is similar to that of measured on $\mathrm{ZnO}$ films grown by PLD. Nevertheless, the higher mobility (above $100 \mathrm{~cm}^{2} / \mathrm{Vs}$ ) leading to a resistivity of $10^{-2} \Omega \mathrm{cm}$ for PLD films can only be obtained for optimized PLD process such as a multi step growth, or buffer layers or even with substrates like $\mathrm{ScAlMgO}_{4}$ to reduce the mismatch and increase the crystallite size [20].

The plot of $\ln (\rho)$ versus the reciprocal temperature for a $\mathrm{ZnO}$ thin film grown at $700{ }^{\circ} \mathrm{C}$ is presented in Fig. 5 and indicates an Arrhenius law versus temperature in the $230-300 \mathrm{~K}$ range. The activation energy $\left(E_{\mathrm{a}}\right)$ is determined from the slope of the linear region of the plot (inset in Fig. 5) and is $11.7 \mathrm{meV}$. From this activation energy, a donor level located at $E_{\mathrm{D}}=2 E_{\mathrm{a}}=0.024 \mathrm{eV}$ below the bottom of the conduction band can be deduced.
A clear deviation from the Arrhenius law is observed when the temperature is below $230 \mathrm{~K}$. This suggests that transport properties could not be anymore correctly described by thermally activated conduction. Classically, it is generally assumed that the transport properties of such films can be described at low temperature range by the variable range hopping model (VRH), in which electrons hop between deep donor states [21]. In this model, the resistivity follows the expression:

$\rho=\rho_{0} \times \exp \left[\left(\frac{T_{0}}{T}\right)^{1 / 4}\right]$

where $\rho_{0}$ is a function of $T^{1 / 2}$ and $T_{0}$ is constant. According to Eq. (1), it follows that $\ln \left(\rho T^{-1 / 2}\right)$ is proportional to $T^{-1 / 4}$. This relation is presented in Fig. 6. The data can be correctly adjusted by a linear variation in the low temperatures domain which confirms the VRH model at low temperature (below the Arrhenius activated regime) in the films, in the short range of $150-120 \mathrm{~K}$. These transport properties

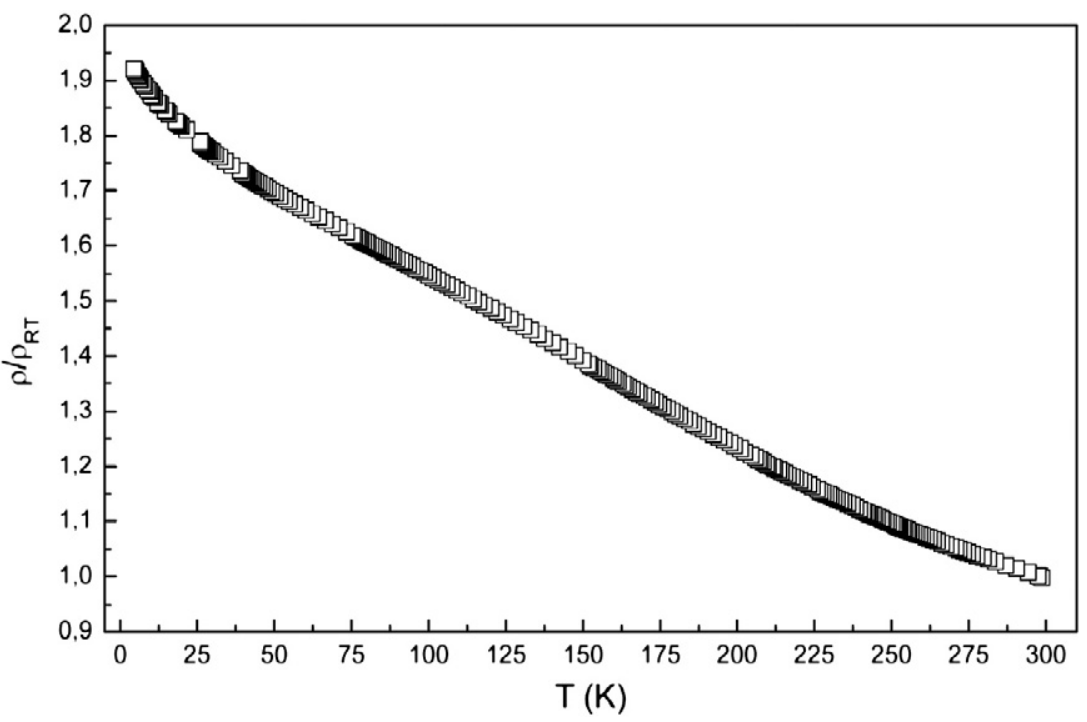

Fig. 4. Temperature dependence of the normalized electrical resistivity $\left(\rho(T) / \rho_{300 \mathrm{~K}}\right)$ for the $\mathrm{ZnO}$ thin films grown on $c$-cut sapphire substrates at $700{ }^{\circ} \mathrm{C}$. 


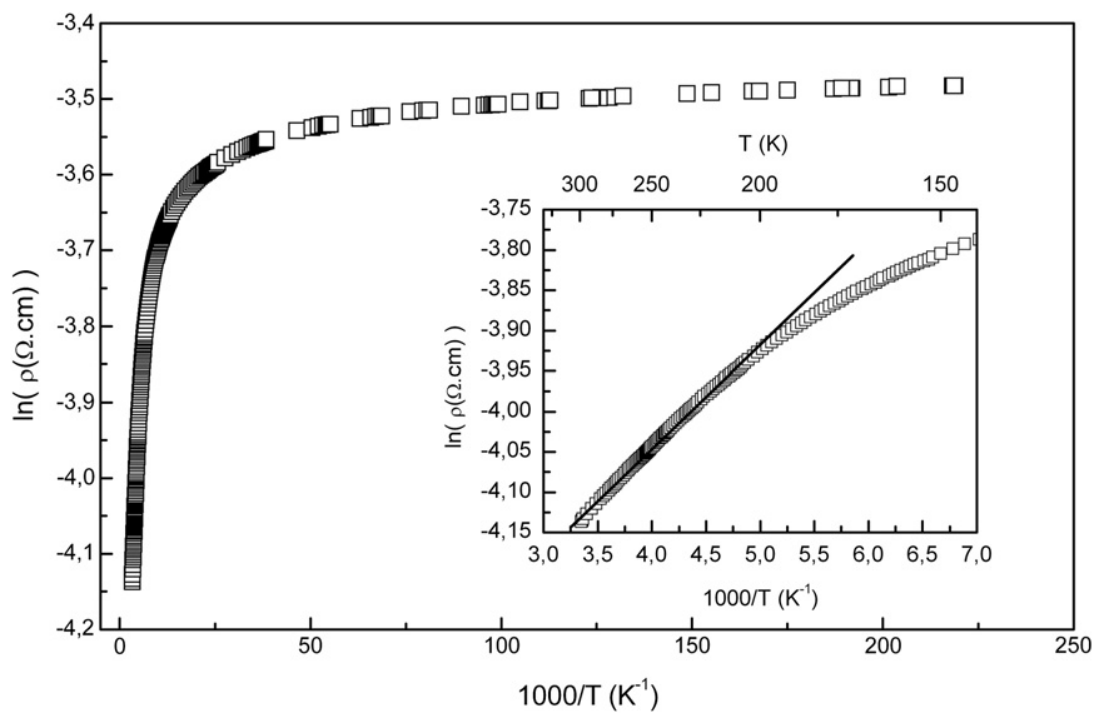

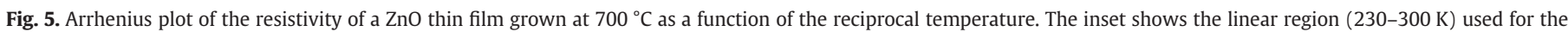
determination of the activation energy.

of $\mathrm{ZnO}$ thin films are not PED specific since the transition between the classical thermally activated conduction to the variable range hopping mechanism has already been reported for $\mathrm{ZnO}$ films prepared by other methods and not fully explained. However, the temperature range in which the VRH model holds is quite reduced, and the temperature domain ranging from 150 to $230 \mathrm{~K}$ cannot be explained by the VRH model or the Arrhenius law. Such temperature dependent resistivity curve can be originated from the high density of charge carriers observed in these films linked to the oxygen vacancies and/or impurities which induces localized states. A metallic behavior of undoped $\mathrm{ZnO}$ PED film displaying a density of carriers in the $1-3 \times 10^{20} \mathrm{~cm}^{-3}$ has been recently observed [22].

\subsection{Optical properties}

Optical properties of $\mathrm{ZnO}$ thin films grown by PED on $c$-cut sapphire substrate at $700{ }^{\circ} \mathrm{C}$ were studied by absorption measurements in the $190-900 \mathrm{~nm}$ wavelength range. Films are well transparent since the transmittance reaches $90 \%$ in the visible range for wavelength above $400 \mathrm{~nm}$. A strong decrease of the transmittance is also observed around $370 \mathrm{~nm}$ which is consistent with good quality $\mathrm{ZnO}$ thin films [23]. This cut-off of the transmittance corresponds to band to band absorption. The energy gap was obtained by applying the Tauc model to absorption measurements [24]. In this model, the variation of the absorption coefficient $\alpha$ in the strong absorption range $\left(\alpha<10^{4} \mathrm{~cm}^{-1}\right)$ is linked to the energy gap $E_{\mathrm{g}}$ of the material by the following expression:

$\alpha=B \times \frac{E-E_{\mathrm{g}}{ }^{m}}{E}$

In the above equation $E$ is the photon energy, $B$ is a constant and the allowed values for $m$ are $1 / 2$ for an allowed direct transition, 3/2 for a forbidden direct transition and 2 and 3 for an allowed and a forbidden indirect transition respectively. Since $\mathrm{ZnO}$ is a direct band gap semiconductor $(m=1 / 2)$, Fig. 7a shows $(\alpha E)^{2}$ as a function of the photon energy $E$. The extrapolation of the linear region of the curve up to $\alpha=0$ gives the value of $E_{\mathrm{g}}$. From Fig. 7a, the optical band gap of $\mathrm{ZnO}$ thin films grown by PED is $3.28 \mathrm{eV}$. This value is in the same range

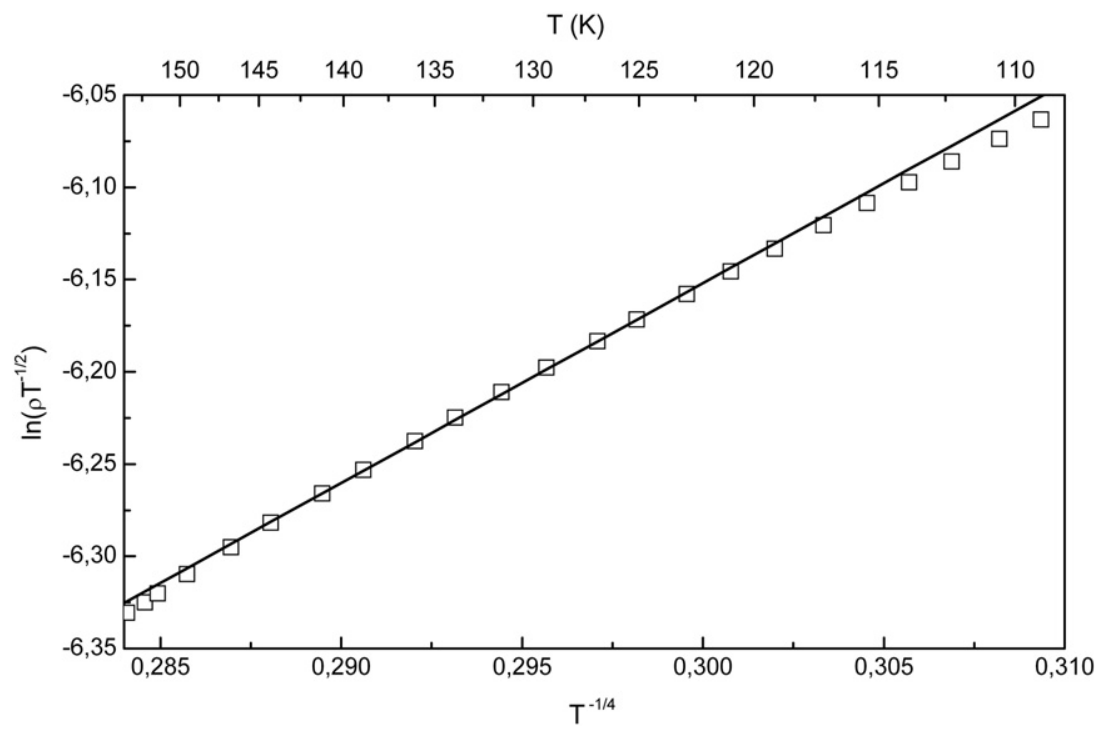

Fig. 6. Variation of $\ln \left(\rho T^{-1 / 2}\right)$ versus $T^{-1 / 4}$ for a $\mathrm{ZnO}$ film grown at $700{ }^{\circ} \mathrm{C}$. The linear fit (solid line) indicates the temperature range where the VRH conduction applies. 

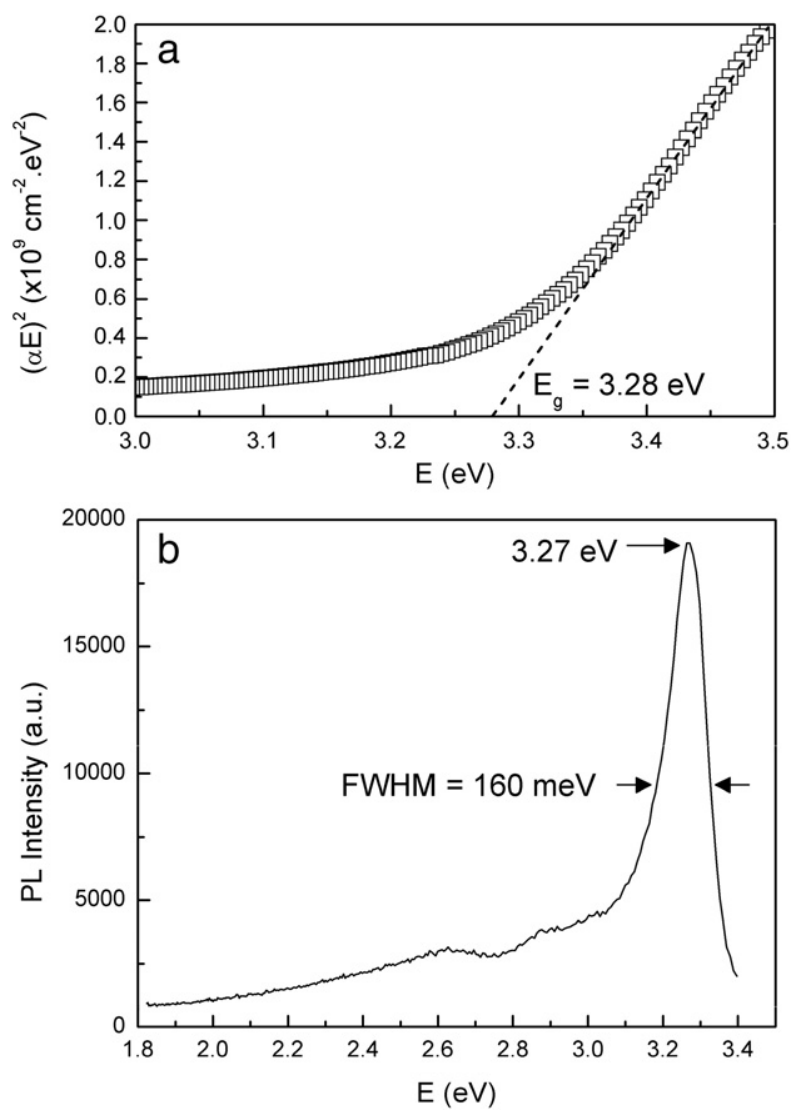

Fig. 7. a) Tauc plot of the absorption coefficient of $\mathrm{ZnO}$ film grown on c-cut sapphire at $700{ }^{\circ} \mathrm{C}$ under $10^{-2}$ mbar Ar. The dash line represents the extrapolation to zero of the linear part of the curve corresponding to the optical band gap of the film. b) Room temperature photoluminescence spectrum of a $\mathrm{ZnO}$ film grown on $c$-cut sapphire by PED at $700{ }^{\circ} \mathrm{C}$.

than that of observed in ZnO films grown by PLD [25] or by other deposition techniques (sputtering, solgel [26]).

Fig. $7 \mathrm{~b}$ shows the normalized photoluminescence spectrum recorded at room temperature. An intense near band edge emission is observed at $3.27 \mathrm{eV}$ with a weak blue-green band (or deep level emission) at about $2.6 \mathrm{eV}$. The lower intensity of the latter indicates a high quality of the $\mathrm{ZnO}$ films grown by PED in $\mathrm{Ar}$ at $700{ }^{\circ} \mathrm{C}$ even without post-deposition thermal treatment under oxygen. The intensity ratio of the near band edge emission to deep-level emissions is about 7 at room temperature. The deep-level emission is usually related to structural defects but in the case of $\mathrm{ZnO}$ films grown on $c$-cut substrate the lattice mismatch plays an important role even for epitaxial thin films grown by PLD [27]. The long tail extending from the near band edge emission, which is usually caused by band structure deformation resulting from lattice deformation [17], is confirmed by the investigation of structural properties (cf. Section 3.2).

In relation to $\mathrm{ZnO}$ films grown on $c$-plane sapphire substrates by PLD [28] the as-deposited PED ZnO films show good optical properties. To improve them, a high temperature annealing treatment in oxygen is probably needed as it is classically performed on $\mathrm{ZnO}$ films grown by various techniques. Nevertheless it was not the aim of this work since the physical properties of the ZnO PED films are compared with those of as-deposited ZnO PLD films. These results therefore indicate that PED is a viable technique for producing high quality epitaxial thin films of $\mathrm{ZnO}$ for optical applications.

Finally, it has to be noticed that this band gap value is slightly lower than the one of bulk $\mathrm{ZnO}(3.3 \mathrm{eV})$. According to the BursteinMoss effect, the high density of charge carriers is supposed to create donor states in the conduction band which limits thermal or optical excitation. As a consequence the band gap value would be supposed to increase (blue shift). As this effect is not observed for our films, the optical absorption edge may be modified by the change of interactions between donors and host crystal. This phenomenon induces a band gap shrinkage evidenced by a shift of the measured optical gap toward the lowest energy. The competitive effects of the Burnstein-Moss shift and the band gap shrinkage has already been observed in heavily defect-doped ZnO films [29].

\section{Conclusions}

In this work, we have demonstrated the possibility of growing dense, smooth, stoichiometric and crystalline zinc oxide films by the pulsed electron beam deposition method using a channel-spark discharge as the pulsed electron source. These $\mathrm{ZnO}$ films obtained by PED were found to grow epitaxially on $c$-cut sapphire substrates at $700{ }^{\circ} \mathrm{C}$, with the classical epitaxial relationship also observed for $\mathrm{ZnO}$ films grown by the well-known PLD method under similar conditions. In the same way, the optical and electrical properties of PED and PLD $\mathrm{ZnO}$ films are very similar.

These results demonstrate the ability of PED to grow high crystalline quality oxide films showing the same functional properties than films grown by other methods. In addition, the PED technique highlights interesting advantages regarding the experimental process in term of growth rate (10 times higher than that of PLD) and the transfer of the target composition which does not require in the setup the incorporation of oxygen at a controlled pressure.

The PED ZnO films show a resistivity of $10^{-2} \Omega \mathrm{cm}$ at room temperature. Such value classically observed for undoped $\mathrm{ZnO}$ films is due to charge carriers with a relatively high density $\left(3.4 \times 10^{19} \mathrm{~cm}^{-3}\right)$ but with a low mobility $\left(11.53 \mathrm{~cm}^{2} / \mathrm{Vs}\right)$. This could be due to the fact that these films are composed of closely packed hexagonal columns separated by grain boundaries, which are usually thought to be detrimental for high carrier mobility. The temperature dependent resistivity curves of the PED films highlight a semiconducting behavior but cannot be explained by a simple law : at high temperature (above $230 \mathrm{~K}$ ) the resistivity follow the Arrhenius law while the VRH model may be applied at low temperature (below $150 \mathrm{~K})$. The optical band gap of PED ZnO films is close to the bulk value. Nevertheless, more detailed investigations have to be made to explain the shift of the optical edge observed on PED ZnO films according to the density of charge carriers and its consequence on the Burstein Moss effect and/or the band gap shrinkage. PED ZnO films grown at different temperatures appear therefore as good candidates to go deeper on this subject.

\section{Acknowledgements}

The authors are grateful to S. Kilburger for the AFM experiments and to I. Enculescu for the photoluminescence measurements.

\section{References}

[1] G. Müller, M. Konijnenberg, G. Krafft, C. Schultheiss, in: F.C. Matacotta, G. Ottaviani (Eds.), Science and Technology of Thin Films, World Scientific Publ. Co. PET. LTD, 1995.

[2] M. Nistor, N.B. Mandache, J. Perrière, J. Phys. D Appl. Phys. 41 (2008) 165205

[3] M. Nistor, N.B. Mandache, J. Optoelectron. Adv. Mater. 7 (2005) 1619.

[4] S. Tricot, N. Semmar, L. Lebbah, C. Boulmer-Leborgne, J. Phys. D Appl. Phys. 43 (2010) 065301.

[5] S. Tricot, C. Boulmer-Leborgne, M. Nistor, E. Millon, J. Perrière, J. Phys. D Appl. Phys. 41 (2008) 175205

[6] V. Craciun, S. Amirhaghi, D. Craciun, J. Elders, J.G.E. Gardeniers, I.W. Boyd, Appl. Surf. Sci. 86 (1995) 99

[7] U. Ozgur, Ya.I. Alivov, C. Liu, A. Teke, M.A. Reshchikov, S. Dogan, V. Avrutin, S.-J. Cho, H. Morkoc, J. Appl. Phys. 98 (2005) 041301.

[8] D.C. Look, Mater. Sci. Eng. B 80 (2001) 383.

[9] H. Hosono, Thin Solid Films 515 (2007) 6000

[10] A. Ohtomo, M. Kawasaki, Y. Sakurai, Y. Yoshida, H. Koinuma, P. Yu, Z.K. Tang, G.K.L. Wong, Y. Segawa, Mater. Sci. Eng. B 54 (1998) 24

[11] H.L. Porter, C. Mion, A.L. Cai, X. Zhang, J.F. Muth, Mater. Sci. Eng. B 119 (2005) 210. 
[12] L.R. Doolittle, Nucl. Instrum. Methods Phys. Res. Sect. B 9 (1985) 344.

[13] D.S. Williams, F.A. Baiocchi, R.C. Beairsto, J.M. Brown, R.V. Knoell, S.P. Murarka, J. Vac. Sci. Technol. B 5 (6) (1987) 1723.

[14] N. Fujimura, T. Nishihara, S. Goto, J. Xu, T. Ito, J. Cryst. Growth 130 (1993) 269

[15] J. Perrière, E. Millon, W. Seiler, C. Boulmer-Leborgne, V. Craciun, O. Albert, J.C. Loulergue, J. Etchepare, J. Appl. Phys. 91 (2002) 690.

[16] J. Guo, H.L.M. Chang, D.J. Lam, Appl. Phys. Lett. 61 (1992) 3116.

[17] Y. Chen, D.M. Bagnall, H.-J. Koh, K.-T. Park, K. Hiraga, Z. Zhu, T. Yao, J. Appl. Phys. 84 (1998) 3912.

[18] J. Narayan, B.C. Larson, J. Appl. Phys. 93 (2003) 278.

[19] J.D. Albrecht, P.P. Ruden, S. Limpijumnong, W.R.L. Lambrecht, K.F. Brennan, J. Appl. Phys. 86 (1999) 6864.

[20] A. Ohtomo, K. Tamura, K. Saikusa, K. Takahashi, T. Makino, Y. Segawa, H. Koinuma, M. Kawasaki, Appl. Phys. Lett. 75 (1999) 2635
[21] N.F. Mott, J. Non-Cryst. Solids 1 (1968) 1.

[22] M. Nistor, F. Gherendi, N.B. Mandache, C. Hebert, J. Perriere, W. Seiler, J. Appl. Phys 106 (2009) 103710.

[23] E. Millon, O. Albert, J.C. Loulergue, J. Etchepare, D. Hulin, W. Seiler, J. Perrière, J. Appl. Phys. 88 (2000) 6937.

[24] J. Tauc, in: F. Abelès (Ed.), Optical properties of solids, 1972.

[25] J.N. Zeng, J.K. Low, Z.M. Ren, T. Liew, Y.F. Lu, Appl. Surf. Sci. 197-198 (2002) 362

[26] Y. Natsume, H. Sakata, Mater. Chem. Phys. 78 (2002) 170.

[27] E.S. Shim, H.S. Kang, J.S. Kang, J.H. Kim, S.Y. Lee, Appl. Surf. Sci. 186 (2002) 474.

[28] J.F. Muth, R.M. Kolbas, A.K. Sharma, S. Oktyabrsky, J. Narayan, J. Appl. Phys. 85 (1999) 7884.

[29] A.P. Roth, J.B.N. Webb, D.F. Williams, Phys. Rev. B 25 (1982) 7836. 